\title{
DESIGN OF THE BEAM COMBINATION SECTION FOR STOCHASTIC INJECTION
}

\author{
Ao Liu ${ }^{1}$, David Neuffer, Alan Bross \\ Fermilab, Batavia, Illinois, 60510, USA \\ S.Y. Lee \\ Department of Physics, Indiana University, Bloomington, Indiana, 47405, USA
}

(Dated: July 18, 2013)

\begin{abstract}
The stochastic injection scenario proposed by D. Neuffer in early 1980's [1] features the injection and acceptance of pions into the muon storage ring, and is used in the design and simulation of nuSTORM injection and ring [2,3]. The pions decay to muons in the long decay straight section of the ring, then the secondary muons are accepted by the straight FODO structure. The critical design element in this scenario is the Beam Combination Section (BCS), which serially includes a defocusing quadrupole, a bending dipole, and a focusing quadrupole. This paper describes in detail the the design of such a BCS and the matching from this BCS to the downstream side of magnetic horn used to collect the pions after target.
\end{abstract}

Key words. nuSTORM, Stochastic injection, Muon storage ring, Neutrino factory

\section{Introduction}

The possibility of using a muon decay ring with long straight sections to study neutrino interactions has been recognized and first proposed by Neuffer [1]. The "neutrinos from STORed Muons"(nuSTORM) facility is based on the idea that this decay ring can produce an extremely well understood neutrino beam for oscillation physics and neutrino cross section measurements [2]. The stochastic injection scenario avoids using a separate decay channel and fast kickers, which is very cost efficient. In this initially proposed scheme, the pions at $5 \mathrm{GeV} / \mathrm{c}$ with a $\pm 0.5 \mathrm{GeV} / \mathrm{c}$ (or possibly centered at $6 \mathrm{GeV} / \mathrm{c}$ with also a $\pm 10 \%$ momentum band for future interest) momentum band produced after the target will be collected by a magnetic horn, and delivered to the BCS through a pion transfer beamline. The BCS functions as a chicane which kicks the pion beam into the ring by a horizontally displaced defocusing quadrupole and a bending magnet, viewed from the $5 \mathrm{GeV} / \mathrm{c}$ reference pion. The layout of nuSTORM beamline is shown in Figure 2.1.

\footnotetext{
${ }^{1}$ aoliu@fnal.gov, also affliated to Indiana University.
} 


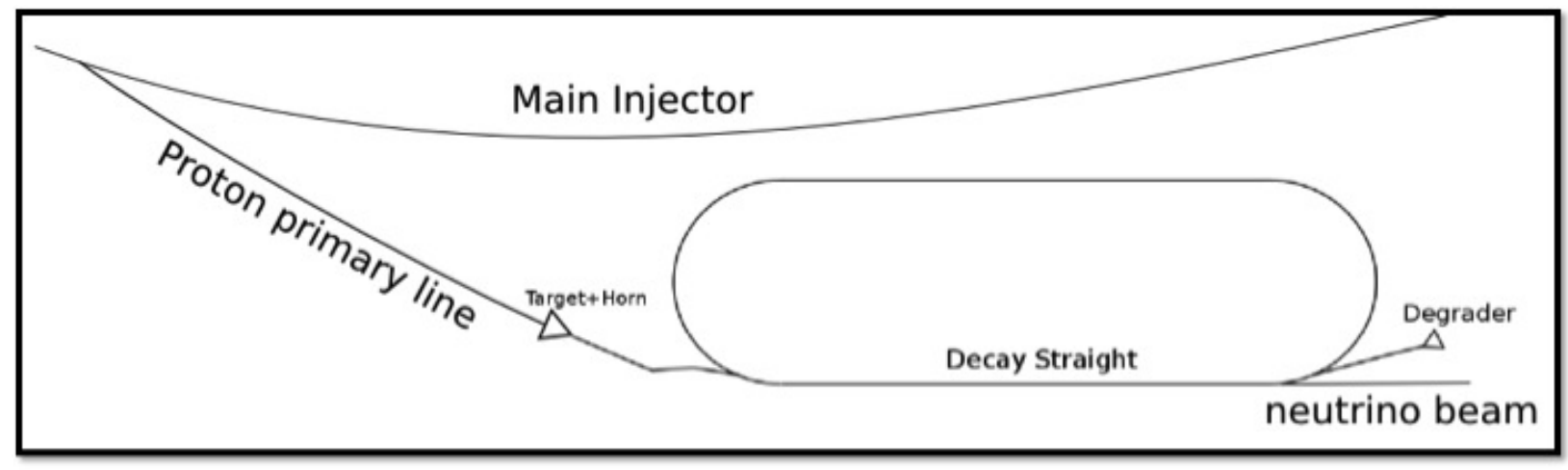

Figure 1.1: The schematic layout drawing of nuSTORM facility at Fermilab.

Once in the ring, the pions will quickly decay to secondary muons which ha a wide momentum distribution centered at $\sim 3.8 \mathrm{GeV} / \mathrm{c}$. In order to accept both the pion beam injected and also as many the muons from pion decay as possible in the decay straight, the decay straight FODO cells need to be designed to incorporate both beams.

In this paper, we will demonstrate the feasibility of this injection scenario by showing the results from the optics design tools MADX [4] and OptiM [5] plus the tracking tool G4Beamline [6].

\section{Optics Design for nuSTORM Injection}

Because stochastic injection uses the muons from pion decay in the injection straight of the ring, the pions will need to proceed until extracted by a simple mirror image of the BCS at the end of injection straight (extraction BCS). The injection described in this section will be not only the beamline for pions before entering the BCS, but also the decay straight section between the BCS and extraction BCS for both pions and muons.

\subsection{FODO Cell Design for nuSTORM Straight Section}

The periodic beta functions for $3.8 \mathrm{GeV} / \mathrm{c}$ muons are determined by an optimization algorithm based on the considerations on beam size, neutrino beam divergence, etc. [2]. The values $\beta_{\max } \sim 30.18 \mathrm{~m}$ and $\beta_{\min }=23.28 \mathrm{~m}$ are chosen and the FODO cell is designed to match these values accurately to avoid beta beat in a series of FODO cell structure. This set of $\beta$ functions, however, can not be used directly to match the pion parameters for the injection. Considering the difference of rigidity of the two beams, which are 12.6754 and $16.6782 \mathrm{~T} \cdot \mathrm{m}$ for $3.8 \mathrm{GeV} / \mathrm{c} \mu$ and $5 \mathrm{GeV} / \mathrm{c} \pi$ (or 20.0138 for $6 \mathrm{GeV} / \mathrm{c} \pi$ ), respectively, the effective focusing coefficients $K_{1}$ differ between the two beams since we will keep constant currents in the magnets. 

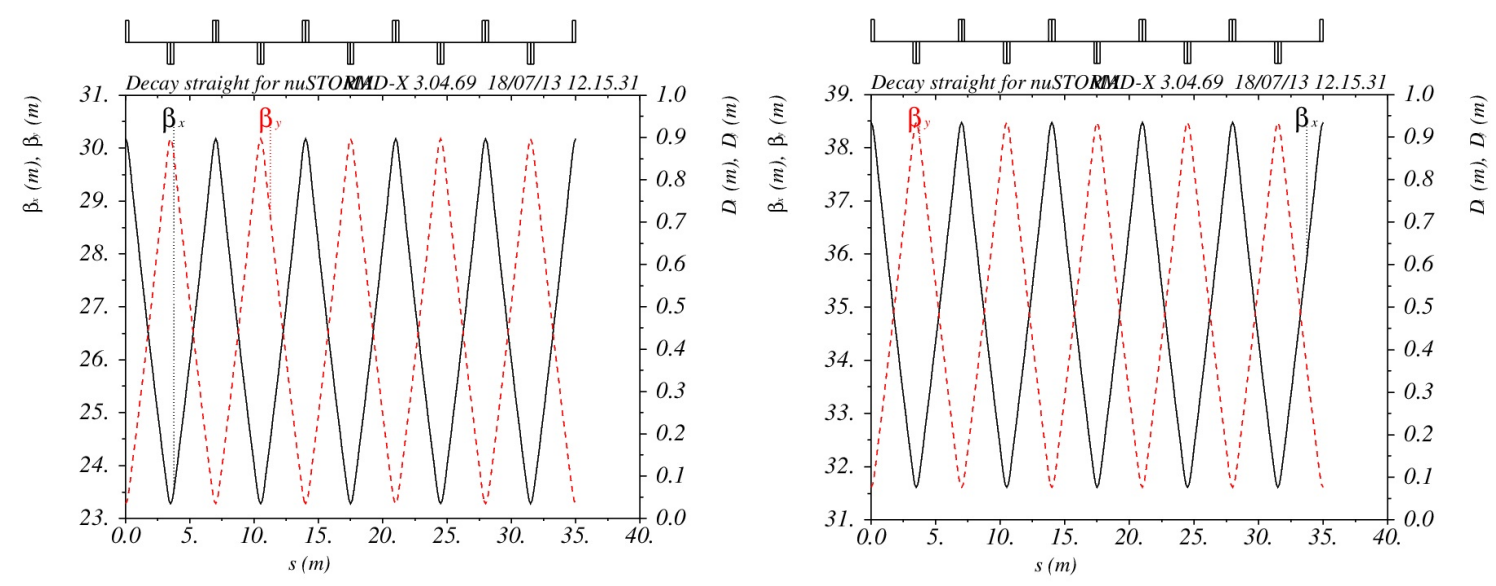

Figure 2.1: Comparison between $\beta$ functions for $3.8 \mathrm{GeV} / \mathrm{c}$ muon (left) and $5 \mathrm{GeV} / \mathrm{c}$ pion (right) in the same FODO cells.

In order to have more useful pion decays, we need to find another set of periodic $\beta$ functions for the same decay FODO straight to transport the 5 (or 6) $\mathrm{GeV} / \mathrm{c}$ pion beam. This can be done via MADX conveniently. Since as a convention, MADX uses $K_{1}=B_{1} / B \rho$ rather than the gradient $B_{1}=\frac{\partial B_{y}}{\partial x}$ as the input parameter for a quadrupole, the values for $K_{1}$ change for the two beams. Therefore, only a scaling factor is needed to transform the same FODO quadrupole from one beam to another, once the $K_{1}$ for the first beam has been found by the MATCHING module in MADX. An example of setting up $K_{1}$ for $3.8 \mathrm{GeV} / \mathrm{c}$ muons and $6 \mathrm{GeV} / \mathrm{c}$ pions is shown below.

! FOR MU at $3.8 \mathrm{GeV} / \mathrm{c}$;

QbstrFmu: QUADRUPOLE, L=L1, K1=0.158655585567;

QbstrDmu: QUADRUPOLE, L=L1, K1=-0.158655585441;

! For pi at $6 \mathrm{GeV} / \mathrm{c}$;

QbstrFpi6: QUADRUPOLE, L=L1, K1=QbstrFmu->k1*12.6754/20.0138;

QbstrDpi6: QUADRUPOLE, L=L1, K1=QbstrDmu->k1*12.6754/20.0138;

Based on the $K_{1}$ of the pion beam, periodic $\beta$ functions, $\beta_{\max } \sim 38.48 \mathrm{~m}, \beta_{\min }=31.61$ and $\beta_{\max } \sim 45.42 \mathrm{~m}, \beta_{\min }=38.56$ are found by MADX matching module for $5 \mathrm{GeV} / \mathrm{c}$ and $6 \mathrm{GeV} / \mathrm{c}$, respectively, to avoid the beta beat of the pion beam after injection. These parameters will be used as the initial parameters in matching between FODO cell to the BCS. The comparison between $\beta$ functions in the FODO cells for the two particles is shown in Figure ?? 


\subsection{Matching from the FODO cells to the BCS}

\subsubsection{Requirements and strategies}

After a proper FODO cell design is obtained, the processes afterwards need to be done opposite to the muon circulating direction or namely the pion injection direction. A large dispersion value $D_{x}$ is needed thereafter to allow the separation of reference orbits of injecting pion and circulating muon. We consider a minimum orbit separation of approximately 0.5 meters to split the beam pipes. This $D_{x}$ can be created by a combination of a bending dipole and a horizontal-defocusing quadrupole, which are centered along the orbit of the $3.8 \mathrm{GeV} / \mathrm{c}$ reference muon. We name this combination along with a horizontal-focusing quadrupole, and the drift spaces in between the three magnets, the Beam Combination Section (BCS).

With the requirement for a large dispersion value induced by the BCS, we also need to reduce the $\beta$ functions in the BCS to avoid unwanted large beam size. The TWISS parameters of the section continued from the FODO cells to the BCS is shown in Figure 2.2 Once this optics is obtained, we need to find the corresponding effective magnetic field the pions see. This can be done either by tracking the reference pion at 5 or $6 \mathrm{GeV} / \mathrm{c}$, or by manually calculating the reference pion orbit. A schematic drawing of the orbits of the reference muon and pion in the magnets is shown in Figure 2.3 and Figure 2.4.

As seen from Figure 2.3 or Figure 2.4, the reference pion enters the BCS offset from the BCS-defocusing quad center forming two non-right angles with its pole faces at entrance and exit, then it enters the sector dipole non-perpendicularly to the pole face, but its orbit will be combined with the muon's after the dipole. The orbit of the reference muon always is perpendicular to the magnets' pole faces and goes through the centers of their magnetic fields.

\subsubsection{Detailed design steps}

The detailed design steps for the BCS are shown below:

- First, track the 5 or $6 \mathrm{GeV} / \mathrm{c}$ pion in the reverse direction of injecting pion beam or circulating muon beam (i.e. Opposite to the direction shown by the arrows in Figure 2.4). Due to the large momentum deviation $\Delta p / p$, this tracking is accurate only when the PTC_TRACK module [4] is applied. The pion starts from the center of the beamline with no angle deviation from the muon reference orbit, but with a momentum deviation denoted by pt.

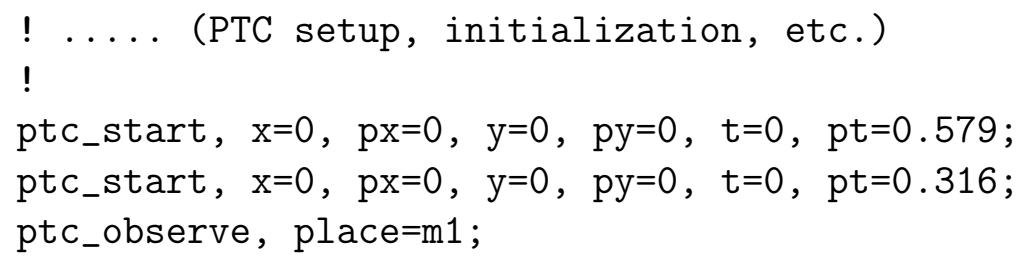




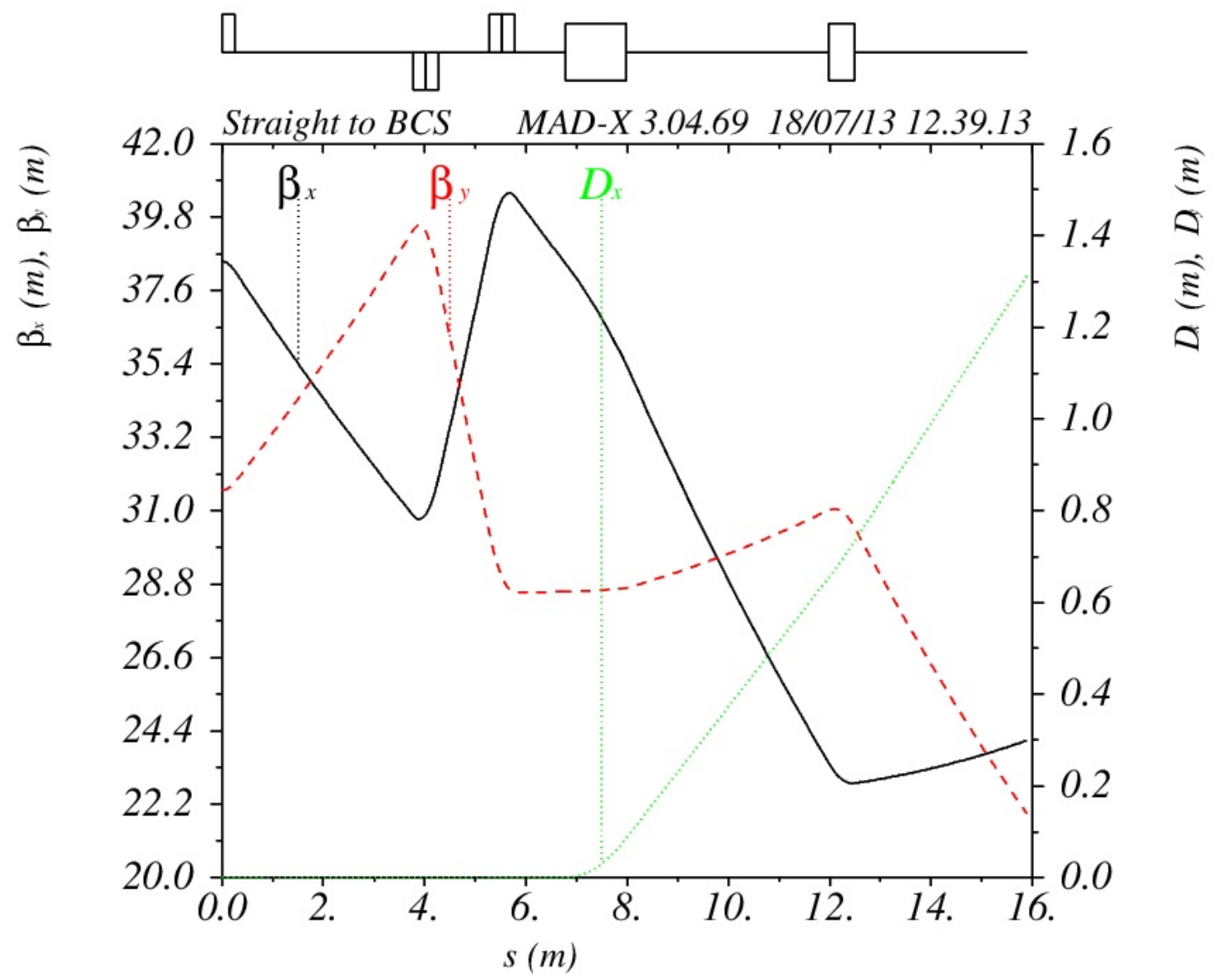

Figure 2.2: The $3.8 \mathrm{GeV} / \mathrm{c}$ muon Optics matched from the first straight FODO cell to the beginning of BCS. From left to right: reverse direction of the injected pions into circulating muon.

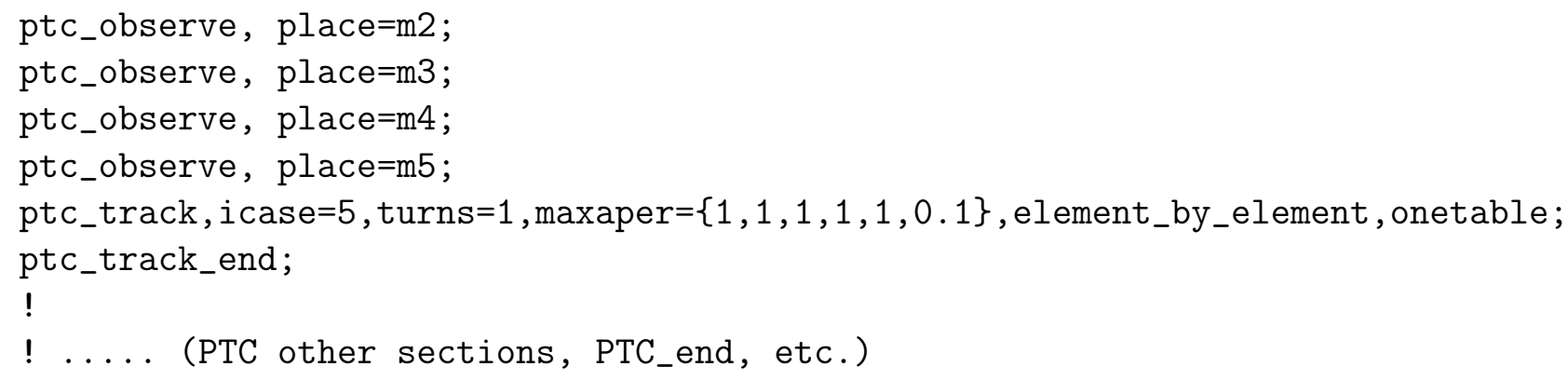

The tracking can also be done and compared with in G4Beamline. Then, confirm that the reference pion is separated by approximately $50 \mathrm{~cm}$, and the beta functions at that point are small enough to avoid larger beam size. Otherwise, redesign the BCS to match the criteria. 


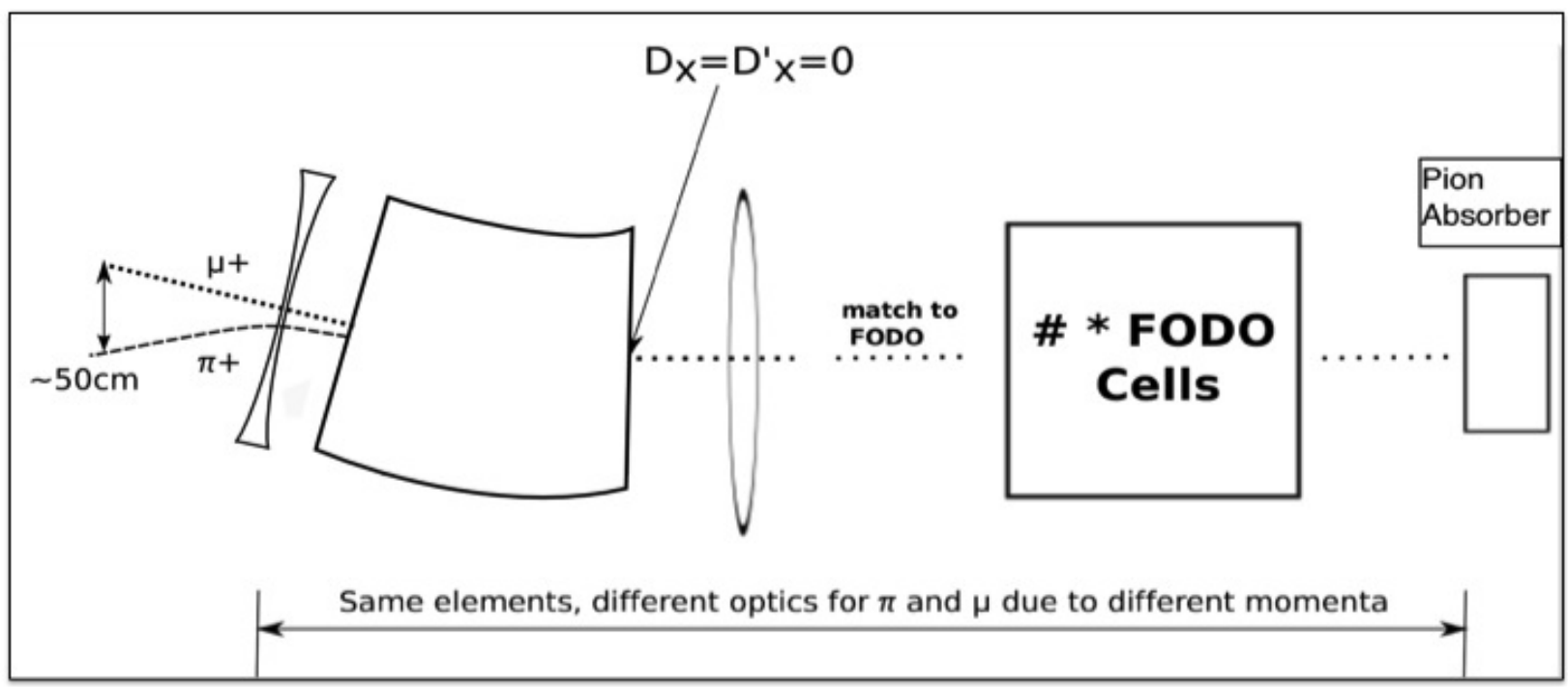

Figure 2.3: Schematic drawing of the reference muon and pion orbits in the magnets. From left to right: the injecting pion direction or namely the circulating muon direction.

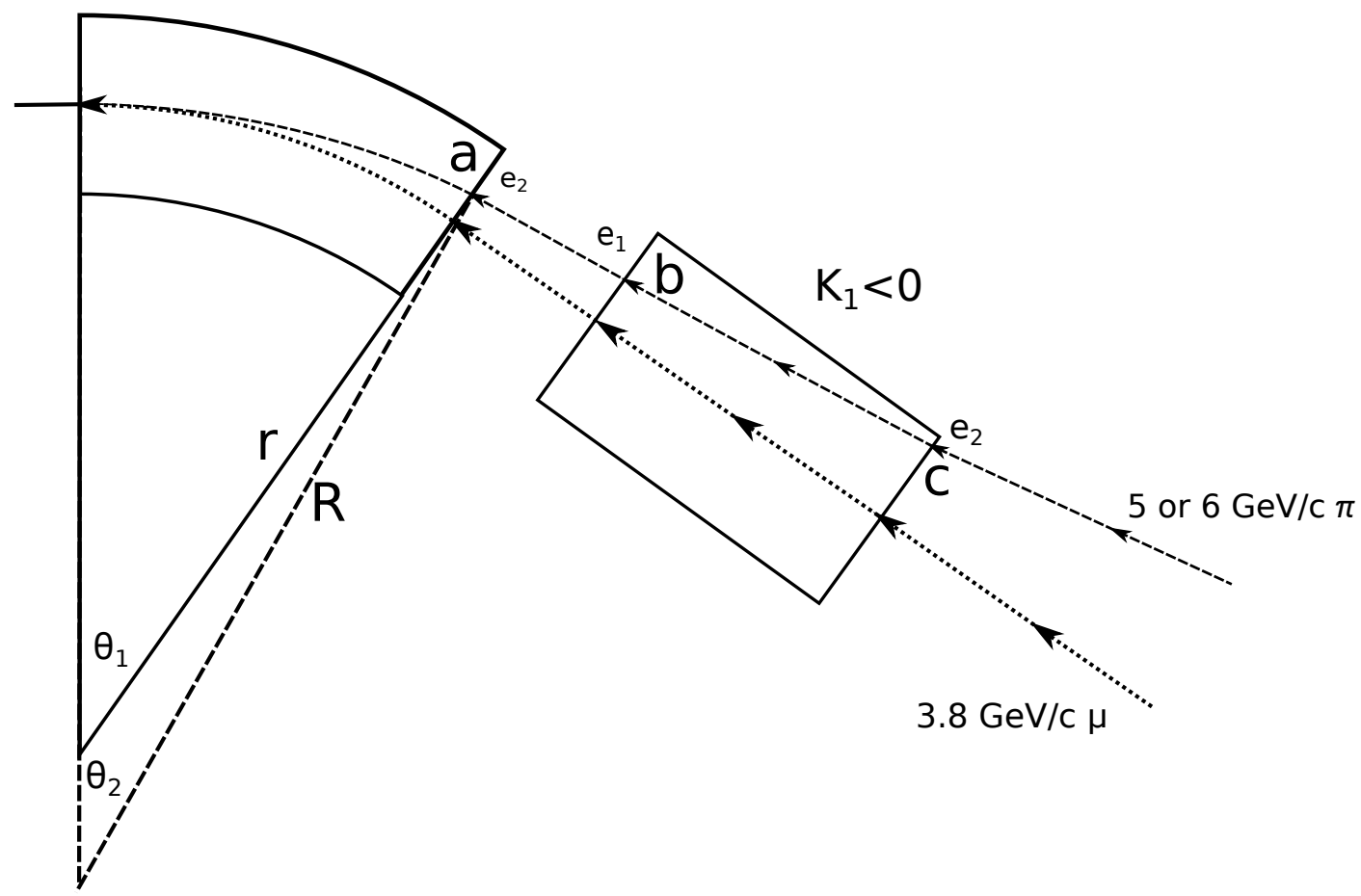

Figure 2.4: Closer view of reference orbits in the BCS magnets. From left to right: reverse direction of the injected pions into circulating muon. 
- Second, read the local horizontal coordinates and momenta of the tracked pion at longitudinal positions, specified by markers in MADX or virtualdetectors in G4Beamline. Three critical points to check are marked by $\mathbf{a}, \mathbf{b}$, and $\mathbf{c}$ in Figure 2.4. Notice that since variable PX in MAD is defined by $p_{x} / p_{0}$ where $p_{0}$ is the reference momentum for the reference particle, which in our case is $3.8 \mathrm{GeV} / \mathrm{c}$ muon, the angle $x^{\prime}$ is obtained by $[(\mathrm{PX} \times 3.8) /(5$ or 6$)]$.

- Third, the sector dipole on the left in Figure 2.4 bends the pion by angle $\theta_{2}$, and has in MADX one more attribute $e^{2}$ which is the exit pole face rotation angle, where $\theta_{2}=\theta_{1}-x_{a}^{\prime}$ and $\mathrm{e} 2=-x_{a}^{\prime}$, according to sign convention in MAD. $x_{a}^{\prime}$ is the horizontal divergence angle at position $\mathbf{a}$. The effective sector dipole length is now $L^{\prime}=R \cdot \theta_{2}$. The basic geometry gives the relation that

$$
\begin{aligned}
R \sin \theta_{2} & =(r+x) \sin \theta_{1} \\
\cos \theta_{2} & =\frac{R^{2}+(R-r)^{2}-(r+x)^{2}}{2 R(R-r)}
\end{aligned}
$$

where $\mathrm{x}$ is the local horizontal coordinate of the pion at a. Equations (2.1) and (2.2) can be used to calculate $R$ and $L^{\prime}$.

The defocusing quadrupole provides an effective dipole field for pions, plus the quadrupole field. The effective bending angle is $x_{c}^{\prime}-x_{b}^{\prime}$, and the effective length is the path length in the quadrupole, which can be well estimated by $L_{D} / \cos \left(\left(x_{c}^{\prime}+x_{b}^{\prime}\right) / 2\right)$ where $L_{D}$ is the field length of the defocusing quadrupole. Notice the bending angle has the opposite sign from the dipole's. The two pole face rotation angles, e1 and e2, are denoted by $\mathrm{e} 1=+x_{b}^{\prime}$ and $\mathrm{e} 2=-x_{c}^{\prime}$, respectively.

Notice that, due to different conventions, the signs for e1 and e2 both flip when the bending angle $\theta$ is negative in OptiM. A csh SHELL script is written to convert the MADX output to OptiM input [7].

- As a summary, the MADX input below shows one example of these two magnets.

BbcsPi5: sbend, $1:=1.202$, angle:=angleBmu-0.0449, e1=0, e2=-0.0449;

BbcsDpi5: sbend, $1:=0.501$, angle $=-0.0128$, e1 $=0.0449$, e2 $=-0.0577$, $\mathrm{k} 1=\mathrm{QbcsDmu}->\mathrm{k} 1 * 12.6754 / 16.6782$;

where angleBmu is the bending angle for the reference muon, and QbcsDmu->k1 is the $\mathrm{k} 1$ value of the defocusing quadrupole for muons. The corresponding optics for 5 $\mathrm{GeV} / \mathrm{c}$ pion from the straight to the BCS is shown in Figure 2.5

- Finally, after the BCS is designed, match the TWISS parameters to values obtained by fitting at the downstream horn. One criterion is that the dispersion $\mathrm{D}_{\mathrm{x}}$ needs to be matched back to 0 at that point. The whole optics from the horn to the first straight FODO (with the injecting beam direction) is shown in Figure 2.6 


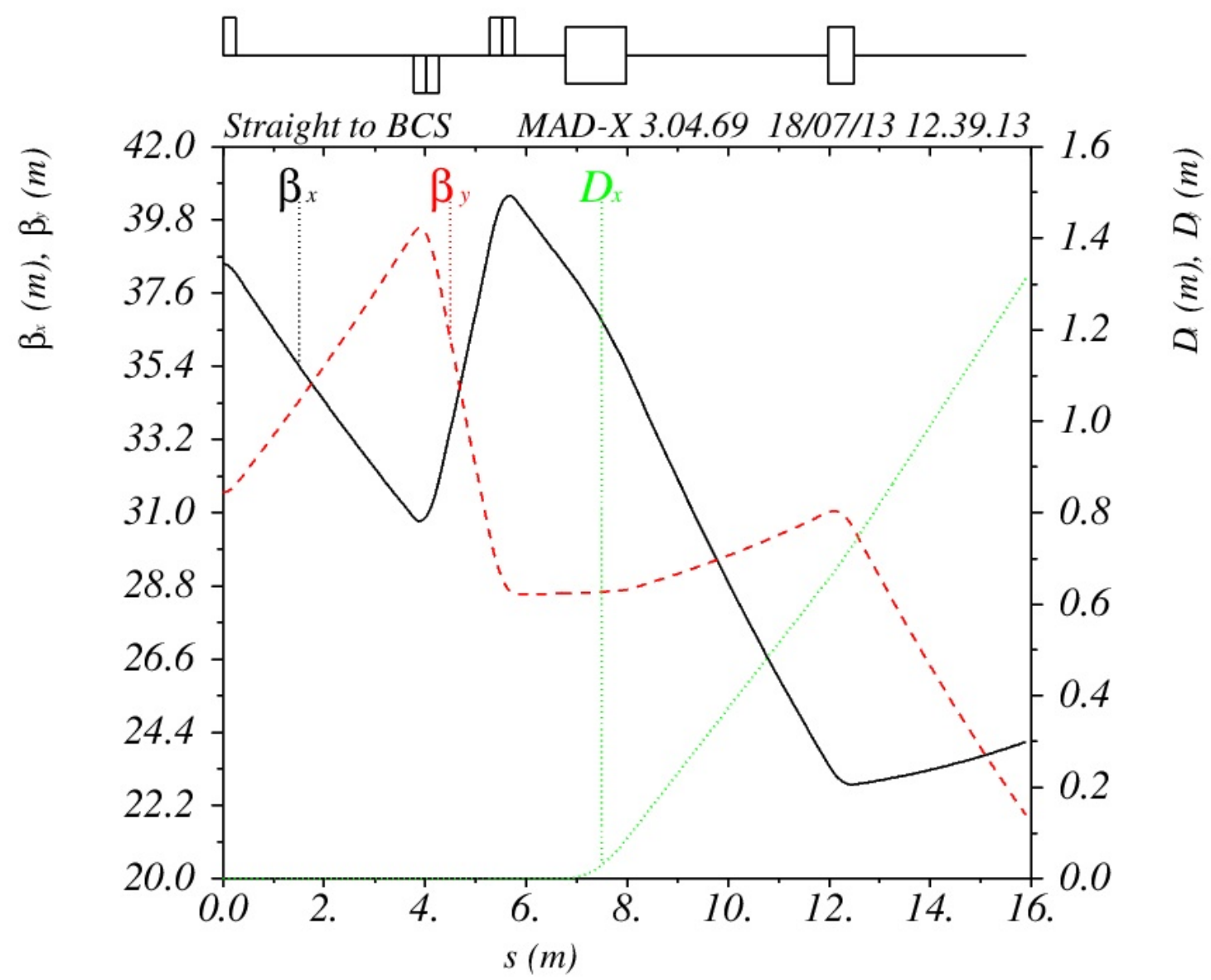

Figure 2.5: The $5 \mathrm{GeV} / \mathrm{c}$ pion optics from the first straight FODO cell to the beginning of BCS. From left to right: the reverse direction of the injecting pion direction or namely the circulating muon direction.

\subsection{Modeling BCS in G4Beamline}

In order to do G4Beamline multi-particle tracking, we need to model the BCS in G4Beamline format. Since G4Beamline constructs real magnets in its world space, it is more realistic if we use the real defocusing quadrupole and sector dipole rather than replacing them with an effective combined-function dipole and a dipole with specially rotated pole faces, respectively. Viewed from the reference injected pion, the defocusing quadrupole is displaced horizontally with a rotation angle with respect to the $\mathrm{y}$ axis, so does the sector dipole. See Figure 2.7 and Figure 2.8 for a BCS visualized in G4Beamline.

Both rotation angles and horizontal displacements can be obtained by the MADX tracking results. The exact tuning can be done manually to center the reference pion in each magnet and beam pipe after the BCS. Both the defocusing quadrupole and sector dipole need to be followed by a correct cornerarc, which bend the reference pion to the right point and 


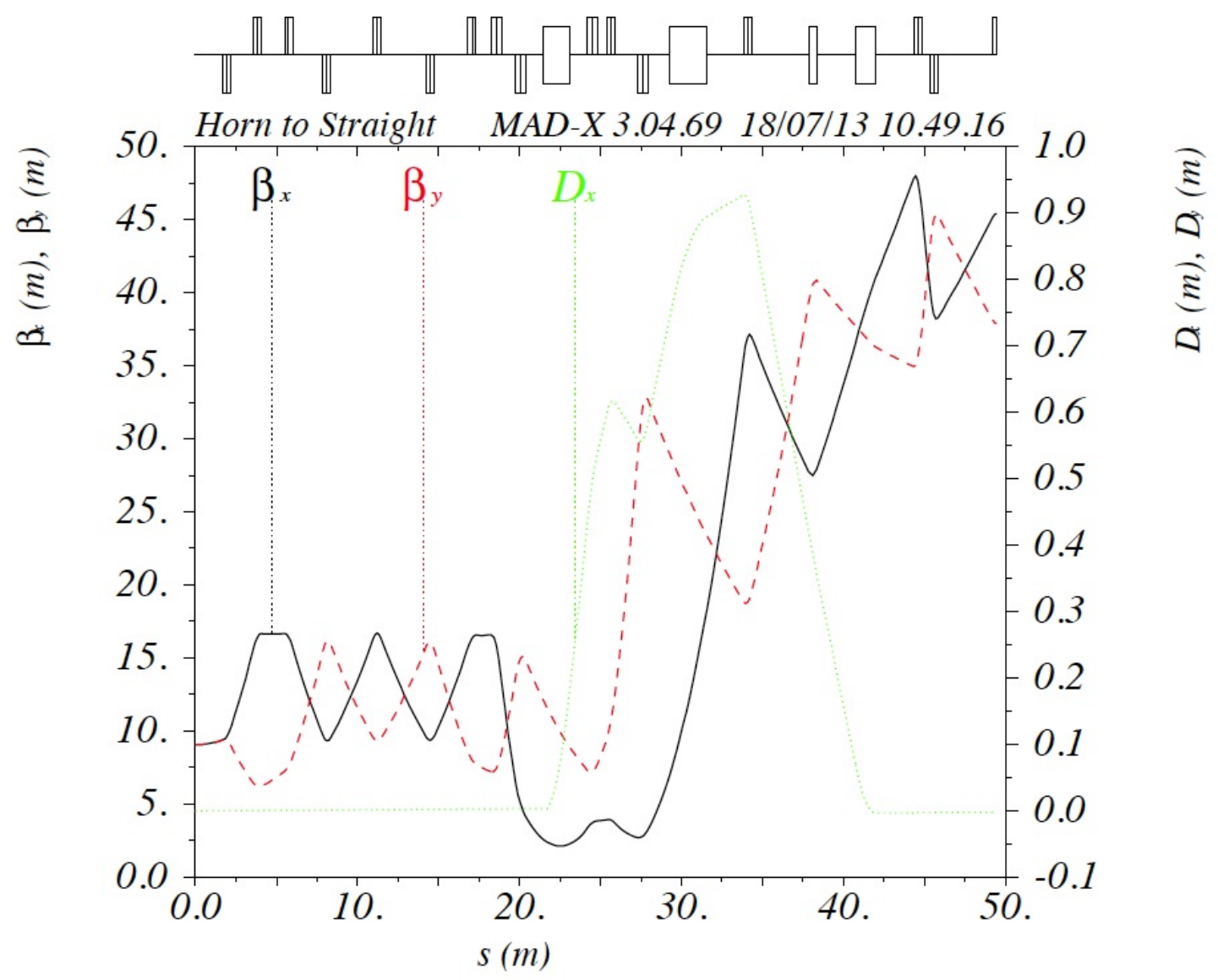

Figure 2.6: The $5 \mathrm{GeV} / \mathrm{c}$ pion optics from the horn to the first decay straight FODO. From left to right: the direction of the injecting pion.

direction. An example is shown below:

\#46 gbbcsdpi62 3803.58 0 8.87714 Angle[deg]=5.21392 Eff.Length [cm]=0 Tilt $[\mathrm{deg}]=0$

\# 47 bbbcsdpi6 $3854.1850 .6 \quad 8.87714 \quad-0.39879100001 .28593$

\# 48 gbbcsdpi61 3854.18 $0 \quad 8.87714$ Angle [deg]=-3.92763 Eff.Length $[\mathrm{cm}]=0$ Tilt $[\operatorname{deg}]=0$

param Lbbbcsdpi6_47=505.991 BENDbbbcsdpi6_47=-1.28593 Bbbbcsdpi6_47=0.887714 param Gbbbcsdpi6_47=-3.98791

genericquad bbbcsdpi6_47 apertureRadius=500 ironRadius $=700$

fieldLength=\$Lbbbcsdpi6_47\

ironLength=\$Lbbbcsdpi6_47 ironMaterial=nusaver gradient=\$Gbbbcsdpi6_47

ironColor $=0.7,0.3,0.3,0.6$ 


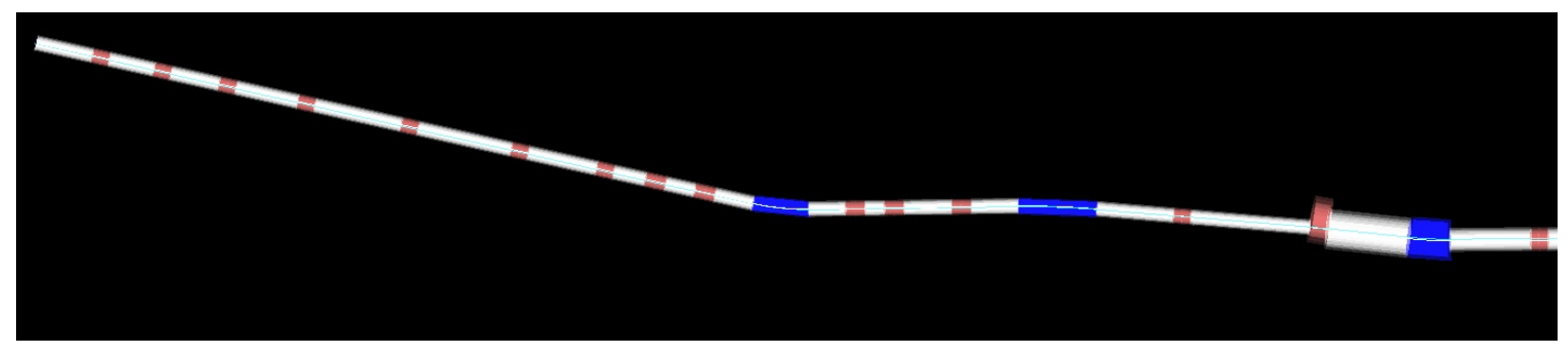

Figure 2.7: The layout of G4Beamline elements from the downstream side of the horn (leftmost) to the BCS focusing quadrupole (rightmost red block)

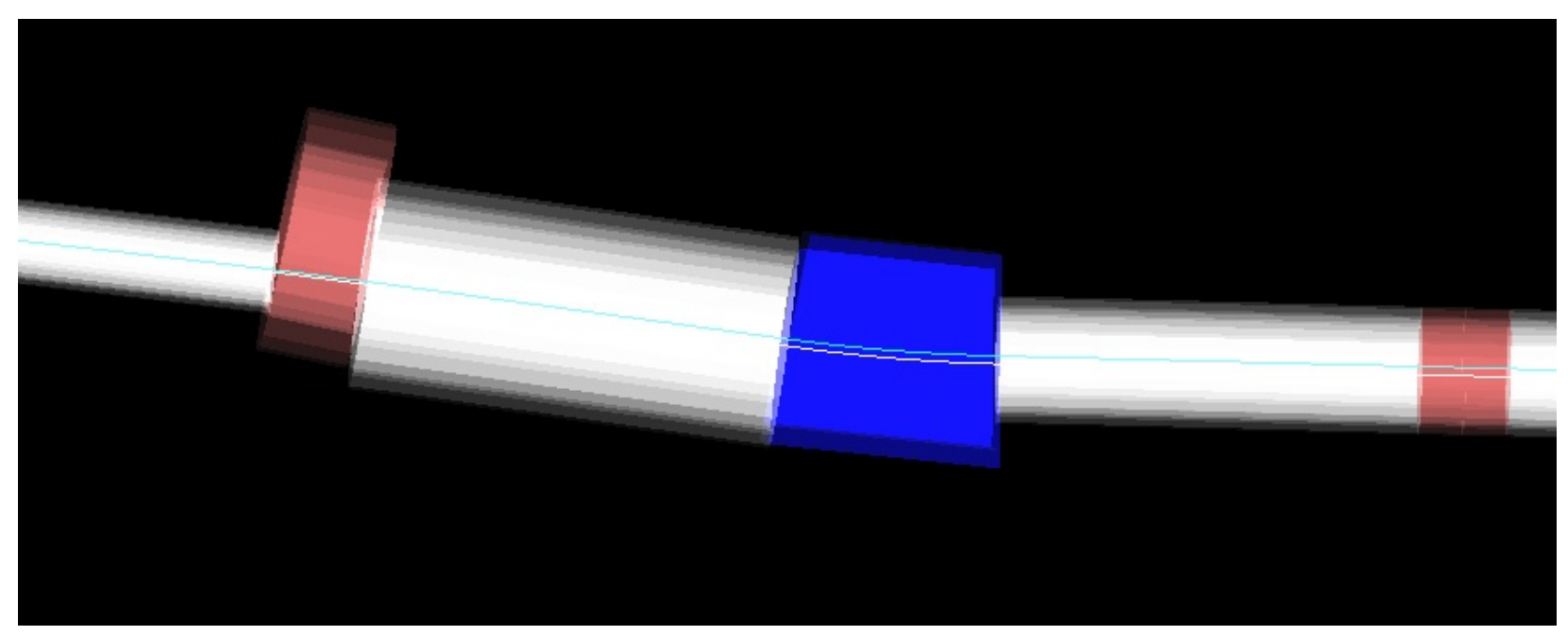

Figure 2.8: Magnified view of the BCS.

place bbbcsdpi6_47 $\mathrm{z}=\$ \mathrm{Z} \mathrm{x}=225$ rotation $=\mathrm{Y}-5.214$ front=1

cornerarc $\mathrm{z}=\$ \mathrm{Z}$ angle $=-1.29$ centerRadius $=20.0138 * 1000 / 0.887714$

param Z=\$Z+\$Lbbbcsdpi6_47-20

\# 49 omm2_4 4094.18240

param Lomm2_4_50=-5+2398.38

tubs omm2_4_50 innerRadius $=\$ I R$ outerRadius $=\$ I R * 1.2$ length $=\$$ Lomm2_4_50

material=nusaver $\backslash$

color $=1,1,1,0.5$

place omm2_4_50 $z=\$ Z$ front $=1$

param Z=\$Z+\$Lomm2_4_50+1.62142

\# 50 gbbcspi62 4094.18 0 -19.8574 Angle[deg]=-3.92763 Eff.Length[cm]=0 Tilt $[\operatorname{deg}]=0$ 
\# 51 bbbcspi6 $4214.57120 .39 \quad-19.85740000000 .84395$

param Lbbbcspi6_52=1203.9 Bbbbcspi6_52=-1.98574 BENDbbbcspi6_52=6.84395

param CRbbbcspi6_52=12.6782*1000/1.98574

param IRbbbcspi6_52=\$CRbbbcspi6_52-\$IR ORbbbcspi6_52=\$CRbbbcspi6_52+\$IR

idealsectorbend bbbcspi6_52 fieldCenterRadius=\$CRbbbcspi6_52

fieldInnerRadius=\$IRbbbcspi6_52 fieldOuterRadius=\$ORbbbcspi6_52

angle $=10.77 \backslash$

fieldHeight $=\$ I R * 2$ ironInnerRadius $=\$ I R b b b c s p i 6 \_52-0.2 * \$ I R$

ironOuterRadius $=\$ O R b b b c$ spi6_52+0.2*\$IR ironHeight=\$IR $* 2.4$ By=\$Bbbbcspi6_52 ।

ironColor $=0,0,1,0.5$ ironMaterial=nusaver

place bbbcspi6_52 $\mathrm{z}=\$ \mathrm{Z} \mathrm{x}=30$ rotation $=\mathrm{Y}-3.92763$

cornerarc $\mathrm{z}=\$ Z$ angle $=6.543$ centerRadius $=20.0138 * 1000 / 1.98574$

param Z=\$Z+\$Lbbbcspi6_52

A csh shell script [8] has been written to convert the OptiM lattice output to G4Beamline input and can be downloaded from the link in the corresponding reference item [8].

\section{Tracking in G4Beamline}

After the above conversion, the BCS and the matching section from the BCS to the first FODO cell magnet will be followed by a series of decay straight FODO cells, which is 147 meters to maintain a high straight-over-arc ratio $\Omega$, provided that the circumference of the ring is fixed at approximately 470 meters.

Take the case where the designed reference pion $\mathrm{p}_{0}=5 \mathrm{GeV} / \mathrm{c}$ for example, the 841,478 pions at the downstream side of the horn from MARS simulation will be taken and converted to G4Beamline ASCII beam format as the initial beam. See Figure 3.1 for the distributions in $\mathrm{X}-\mathrm{Y}$ real space, $\mathrm{X}-\mathrm{X}^{\prime}$ phase space, and momentum histogram, respectively. We use the trackcuts command in G4Beamline to keep only the particles we are interested in. virtualdetectors are placed at longitudinal positions where monitoring on the beam is needed. We first run the simulation with decay process forbidden to check the percentage of pions that survive through the injection, then we allow decay and check the number of muons we obtain at the end of decay straight.

Figure 3.2 show the real, phase space distributions and momentum histogram of the 611,335 pions (72.65\% of the above) detected at the end of the decay straight, without decay processes.

Figure 3.3 shows the real, phase space distributions and momentum histogram of the 216,218 muons (25.70\% of the pions at the horn downstream) detected at the end of the decay straight, with decay processes. Among them, 66,482 muons (7.9\% of the pions at the horn downstream) are within the momentum range $3.8 \pm 10 \% \mathrm{GeV} / \mathrm{c}$, which is the momentum band we are designing the ring to accept. As of our interest, the real, phase space and momentum distribution plots are shown in Figure 3.4. 

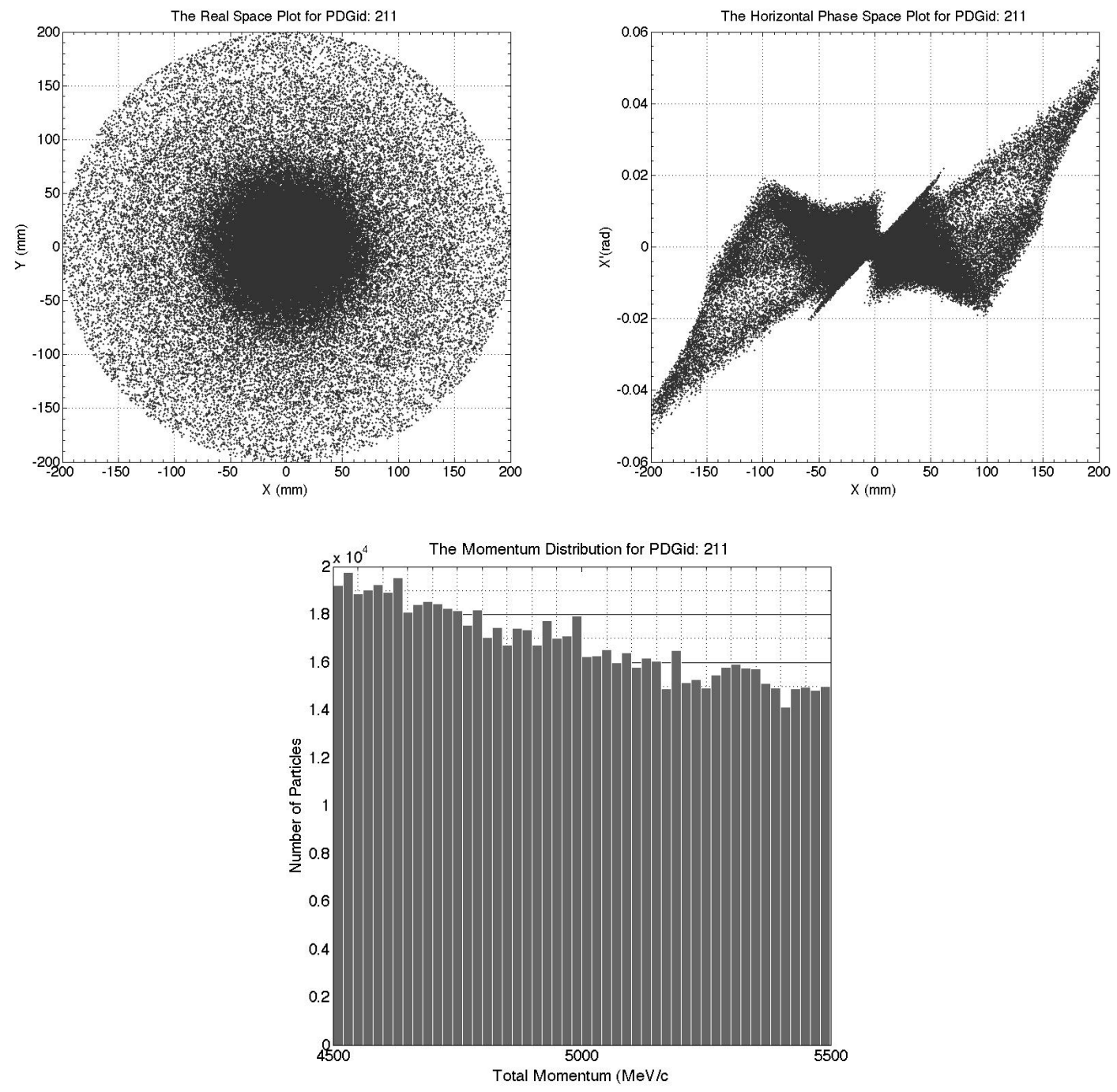

Figure 3.1: X-Y space (upper-left), X-X' space (upper-right) and momentum (lower) distributions of pions at downstream side of the horn.

The projections of the real and phase space distribution onto $\mathrm{x}, \mathrm{x}$ ' and $\mathrm{y}$ axes are shown in Figure 3.5. We see the muons are Gaussian distributed with respect to the center orbit.

The tracking results for $6 \pm 10 \% \mathrm{GeV} / \mathrm{c}$ pions are shown in Appendix. A 

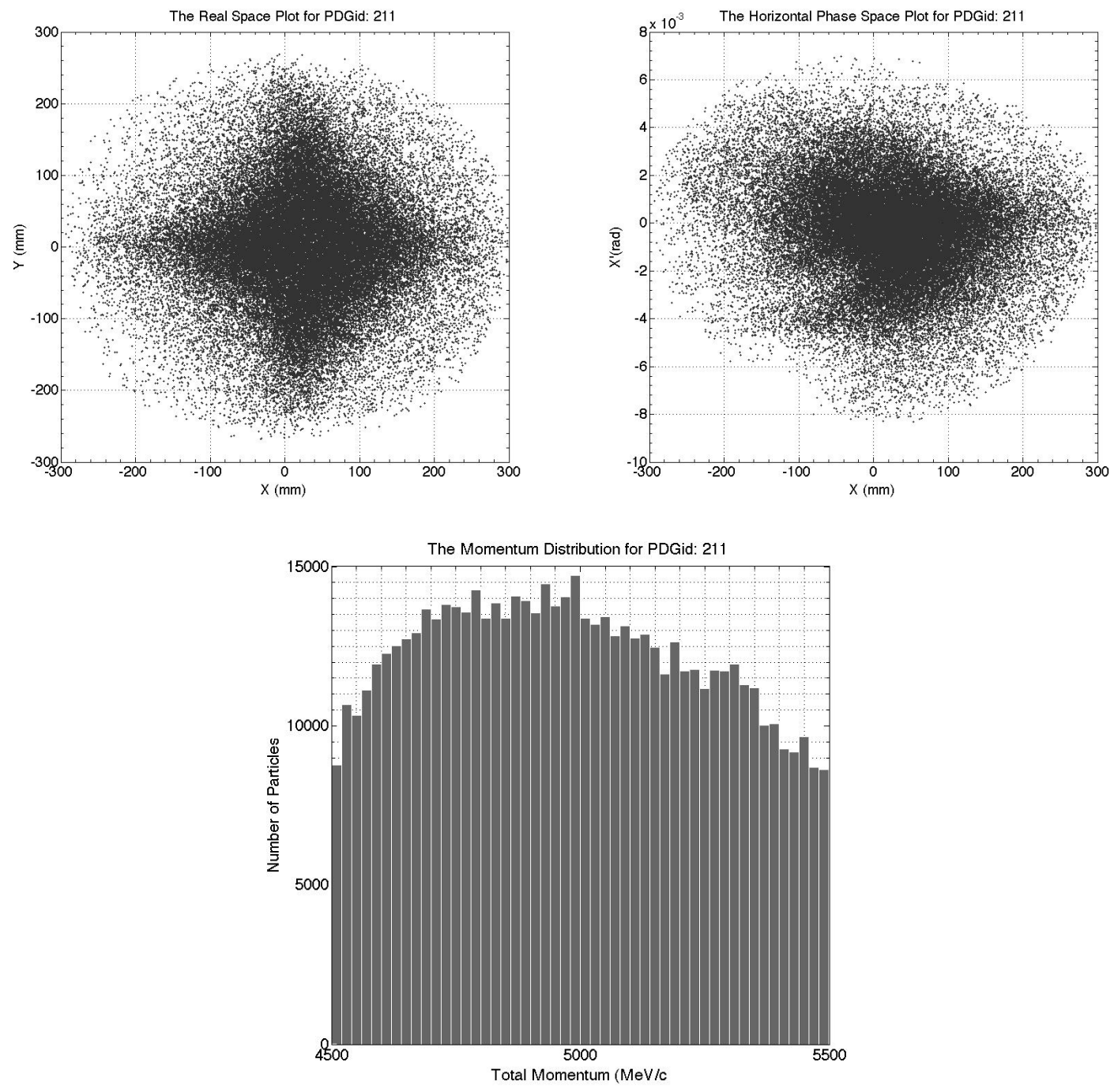

Figure 3.2: X-Y space (upper-left), X-X's space (upper-right) and momentum (lower) distributions of pions at the end of the decay straight. 

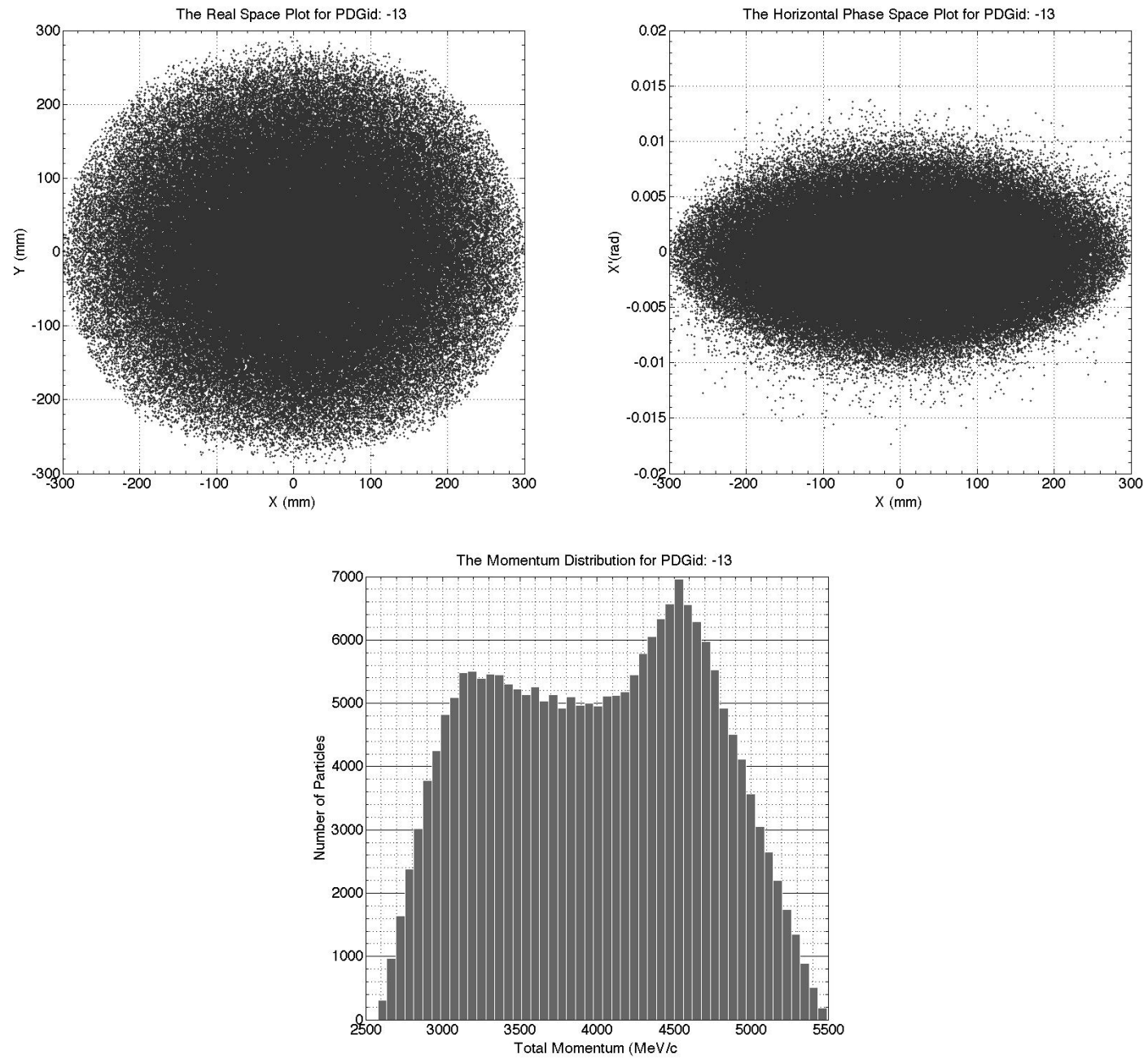

Figure 3.3: $\mathrm{X}-\mathrm{Y}$ space (upper-left), X-X' space (upper-right) and momentum (lower) distributions of muons at the end of the decay straight. 

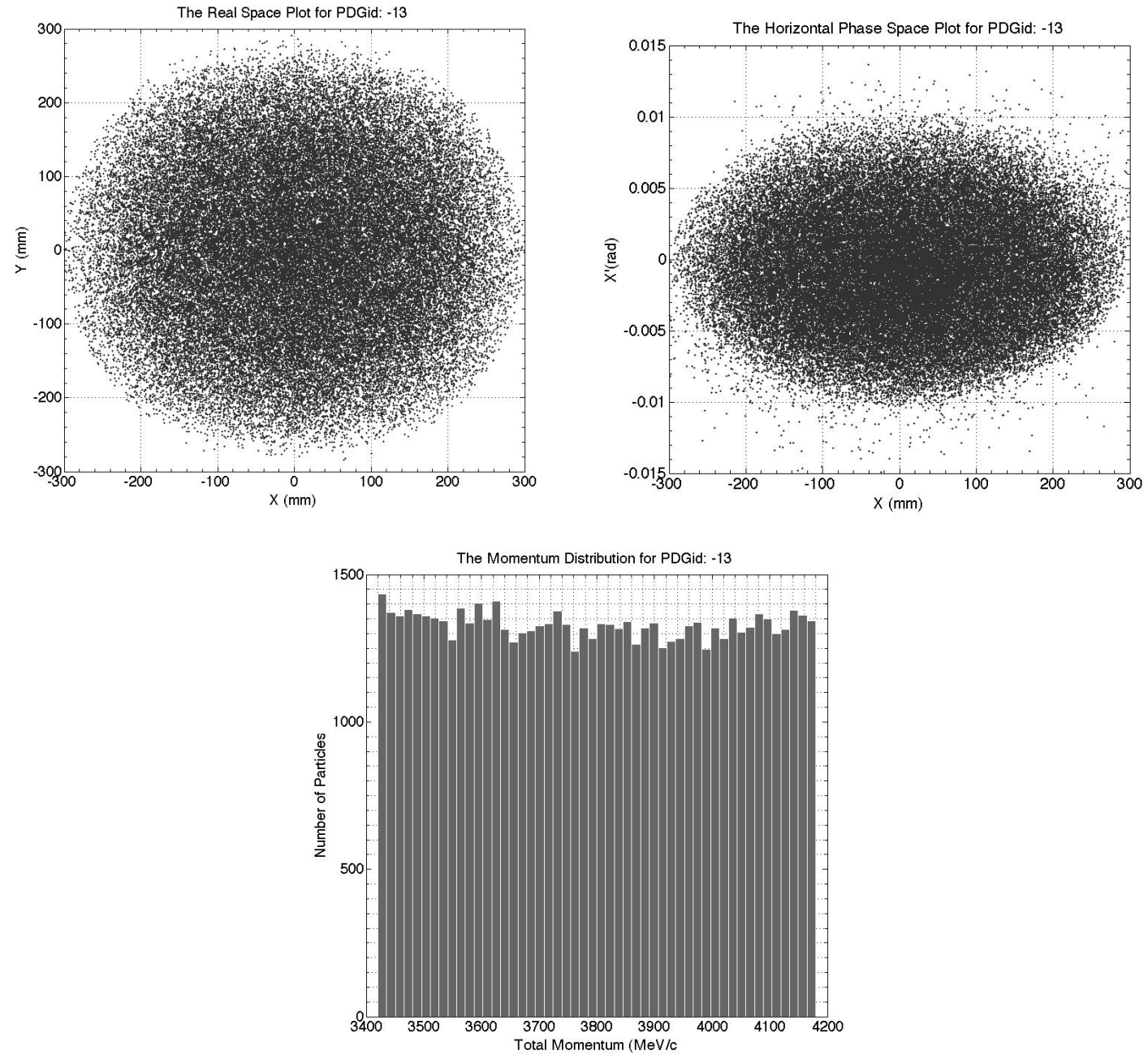

Figure 3.4: $\mathrm{X}-\mathrm{Y}$ space (upper-left), $\mathrm{X}-\mathrm{X}$ ' space (upper-right) and momentum (lower) distributions of muons within $3.8 \pm 10 \% \mathrm{GeV} / \mathrm{c}$ at the end of the decay straight. 

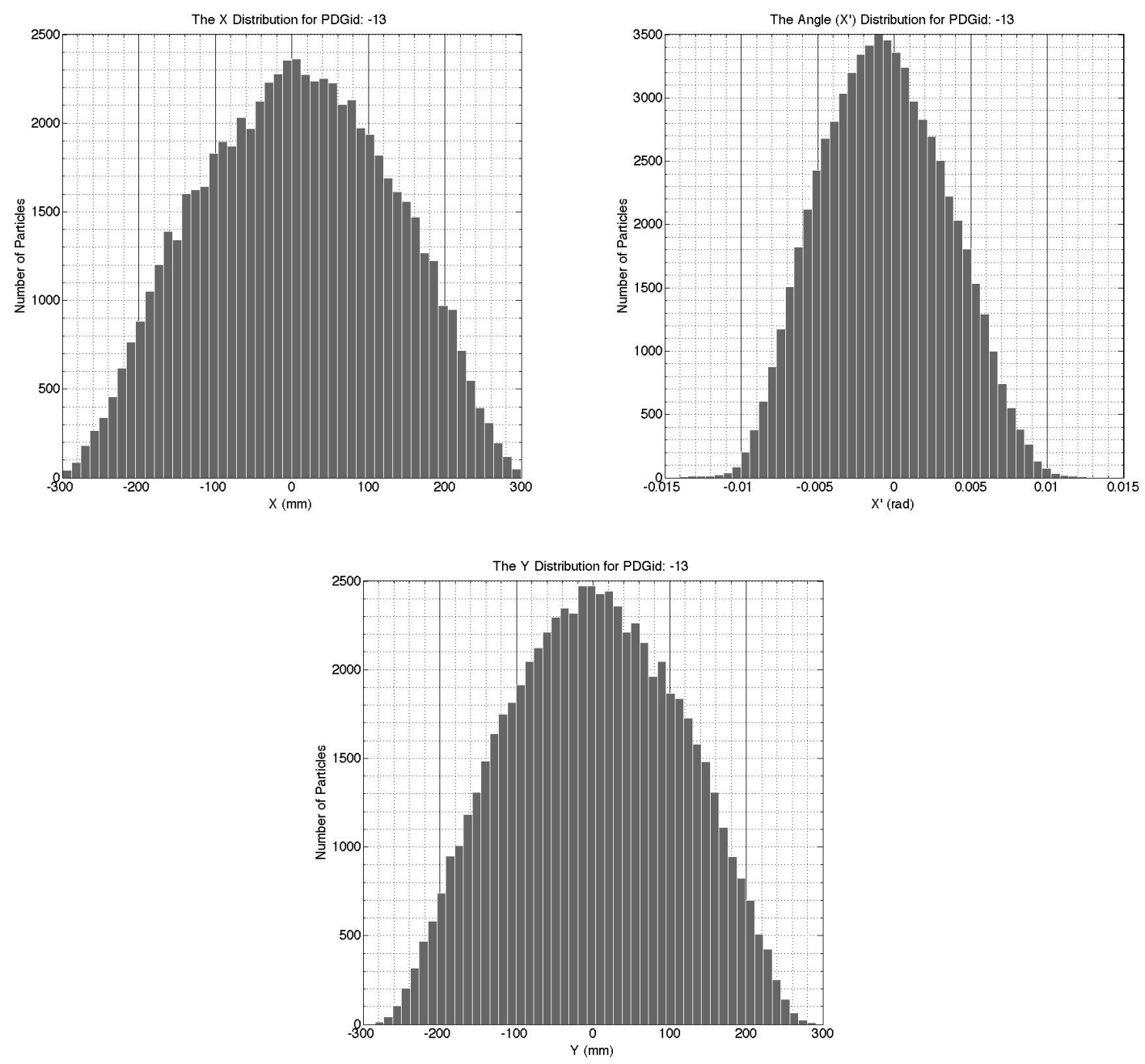

Figure 3.5: Projections of distributions of $3.8 \pm 10 \% \mathrm{GeV} / \mathrm{c}$ muons onto $\mathrm{x}$ axis (upper left), $\mathrm{x}$ ' axis (upper right) and $\mathrm{y}$ axis (lower) 

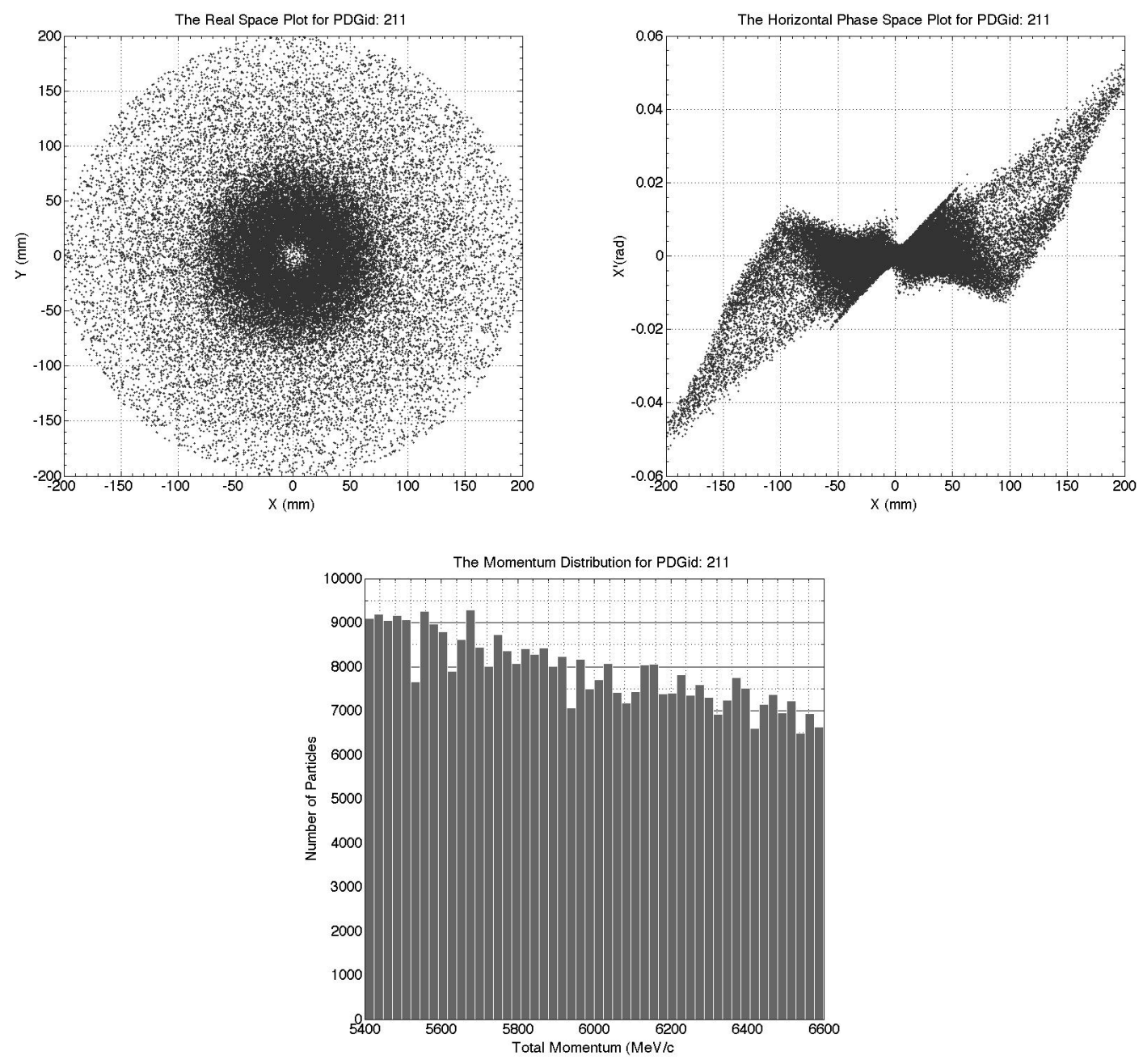

Figure A.1: X-Y space (upper-left), X-X' space (upper-right) and momentum (lower) distributions of the $6 \pm 10 \% \mathrm{GeV} / \mathrm{c}$ pions at horn downstream.

\section{A Appendix: Tracking Results for $6 \pm 10 \% \mathrm{GeV} / \mathrm{c}$ pions}

Figure A.1 shows the real, phase space distributions and momentum histogram of the 395,232 pions at the downstream side of the horn. Figure A.2 shows the position space (x,y), phase space $\left(\mathrm{x}, \mathrm{x}^{\prime}\right)$ distributions and momentum histogram of the 284,873 pions $(72.08 \%$ of the above) detected at the end of the decay straight, without decay processes. Figure A.3 shows the real phase space distributions and momentum histogram of the 95,388 muons $(24.13 \%$ of the pions at the horn downstream) detected at the end of the decay straight, with decay processes. Among them, 23,605 muons (5.97\% of the pions at the horn downstream) are within momentum range $3.8 \pm 10 \% \mathrm{GeV} / \mathrm{c}$, which is the momentum band we are designing 

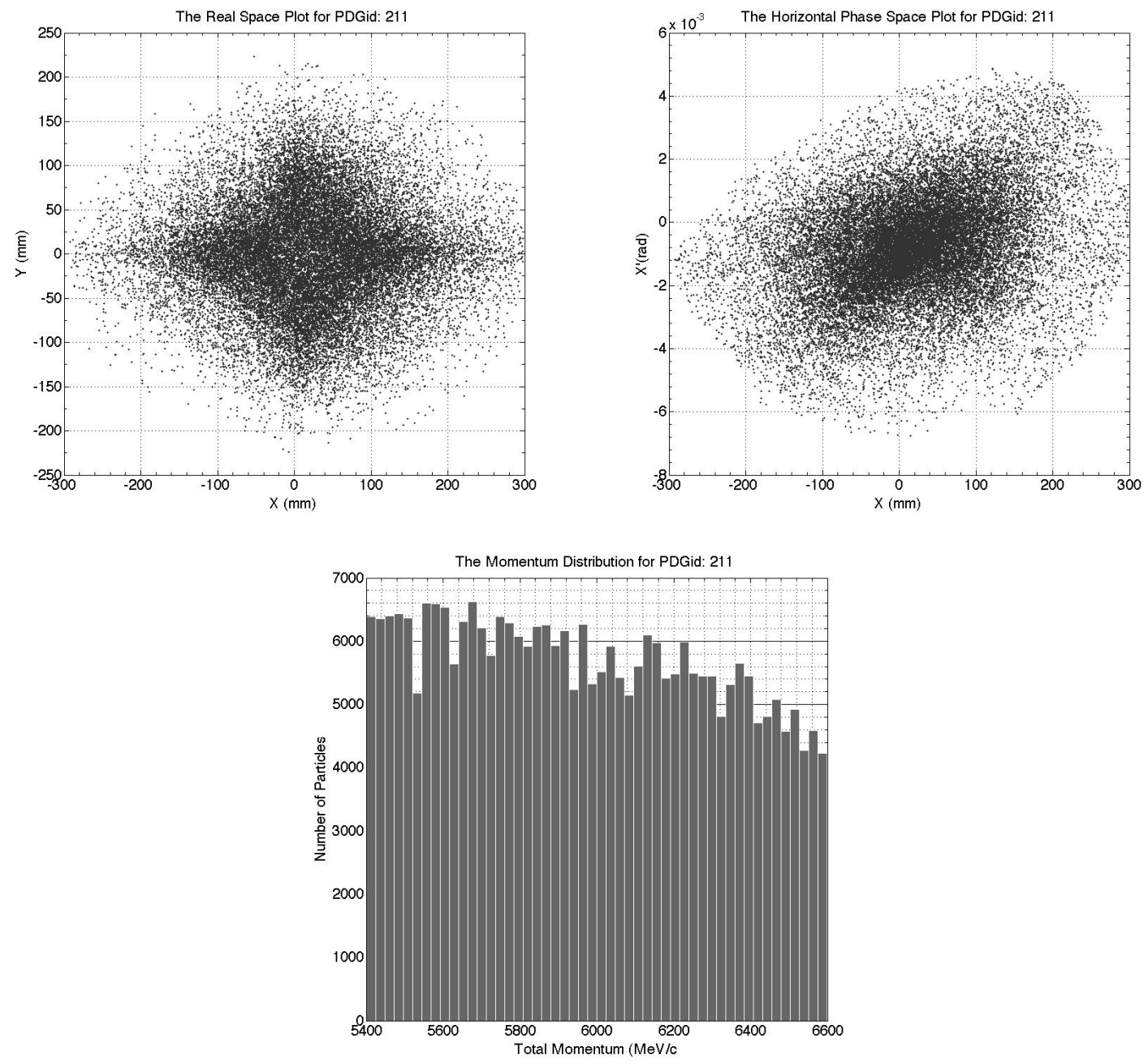

Figure A.2: X-Y space (upper-left), X-X' space (upper-right) and momentum (lower) distributions of pions at the end of the decay straight.

the ring to accept. As of our interest, the real, phase space and momentum distribution plots are shown in Figure A.4. The projections of the real and phase space distribution onto $\mathrm{x}, \mathrm{x}$ ' and $\mathrm{y}$ axes of above muons are shown in Figure A.5. We see the averaged horizontal coordinates of these muons are not as well-centered at $\mathrm{x}=0$ as those in the case where injecting pions are centered at $5 \mathrm{GeV} / \mathrm{c}$. This can be easily understood by considering the fact the most probable momentum of a pion that can generate a $3.8 \mathrm{GeV} / \mathrm{c}$ muon is $5 \mathrm{GeV} / \mathrm{c}$. Because the $5 \mathrm{GeV} / \mathrm{c}$ is not the design momentum of the injection line, the orbit of an on-axis pion of that momentum will not be combined with the orbit of $3.8 \mathrm{GeV} / \mathrm{c}$ muon, but will start betatron oscillation after the BCS. At the longitudinal position where the virtualdetector is, the oscillation is not necessarily at the center. 

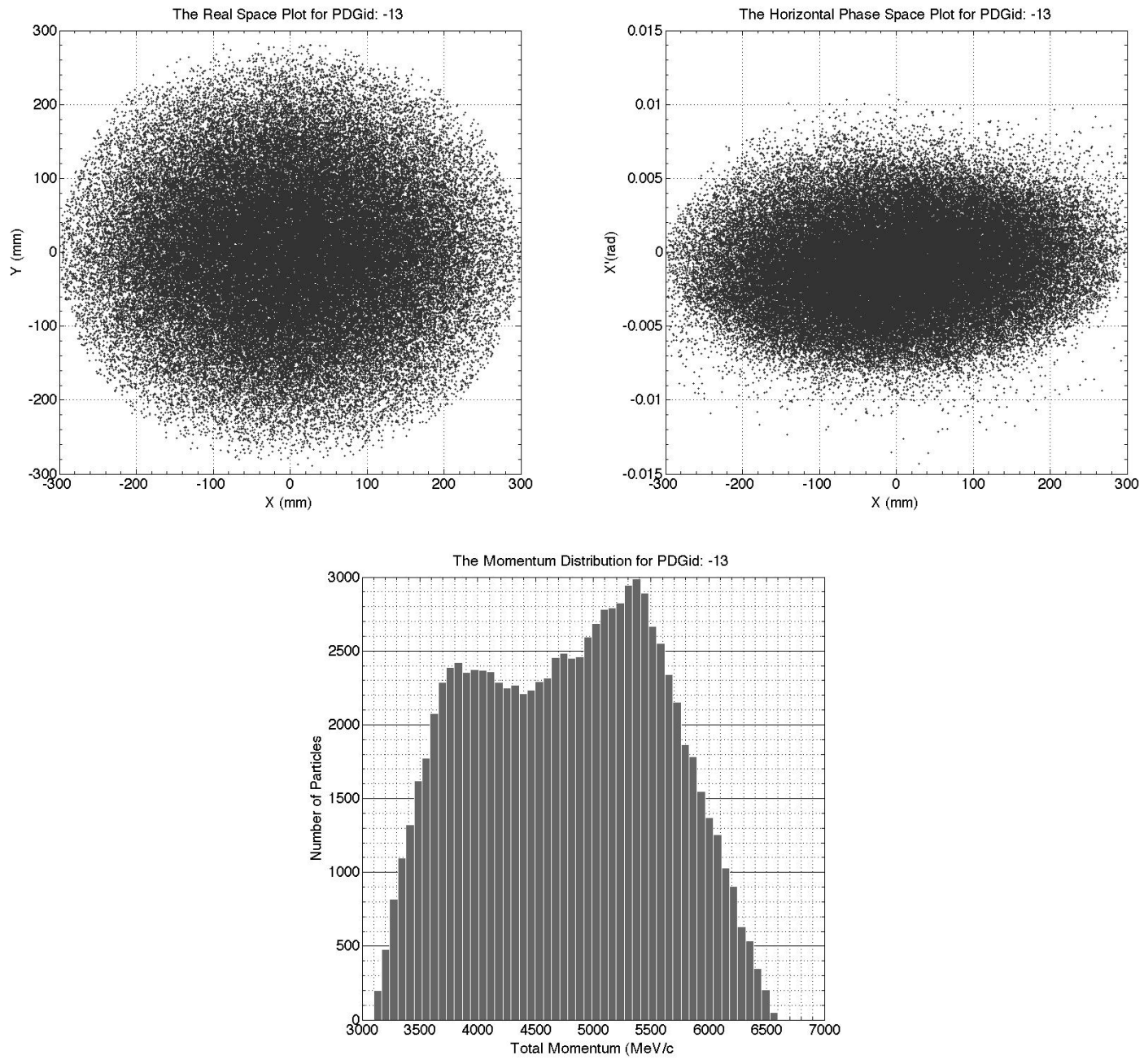

Figure A.3: X-Y space (upper-left), X-X' space (upper-right) and momentum (lower) distributions of muons from $6 \pm 10 \% \mathrm{GeV} / \mathrm{c}$ pions at the end of the decay straight. 

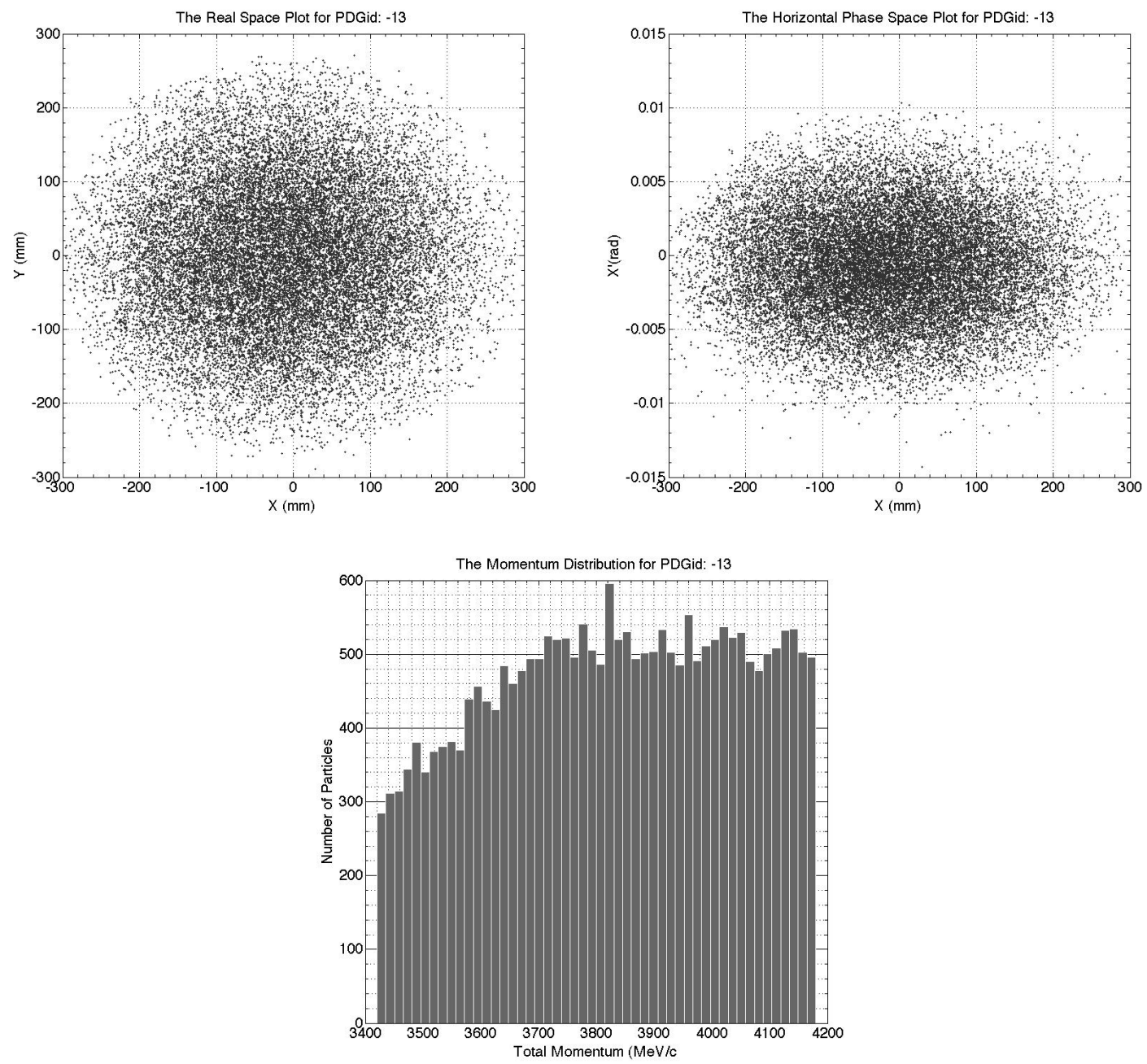

Figure A.4: X-Y space (upper-left), X-X' space (upper-right) and momentum (lower) distributions of muons within $3.8 \pm 10 \% \mathrm{GeV} / \mathrm{c}$ at the end of the decay straight. 

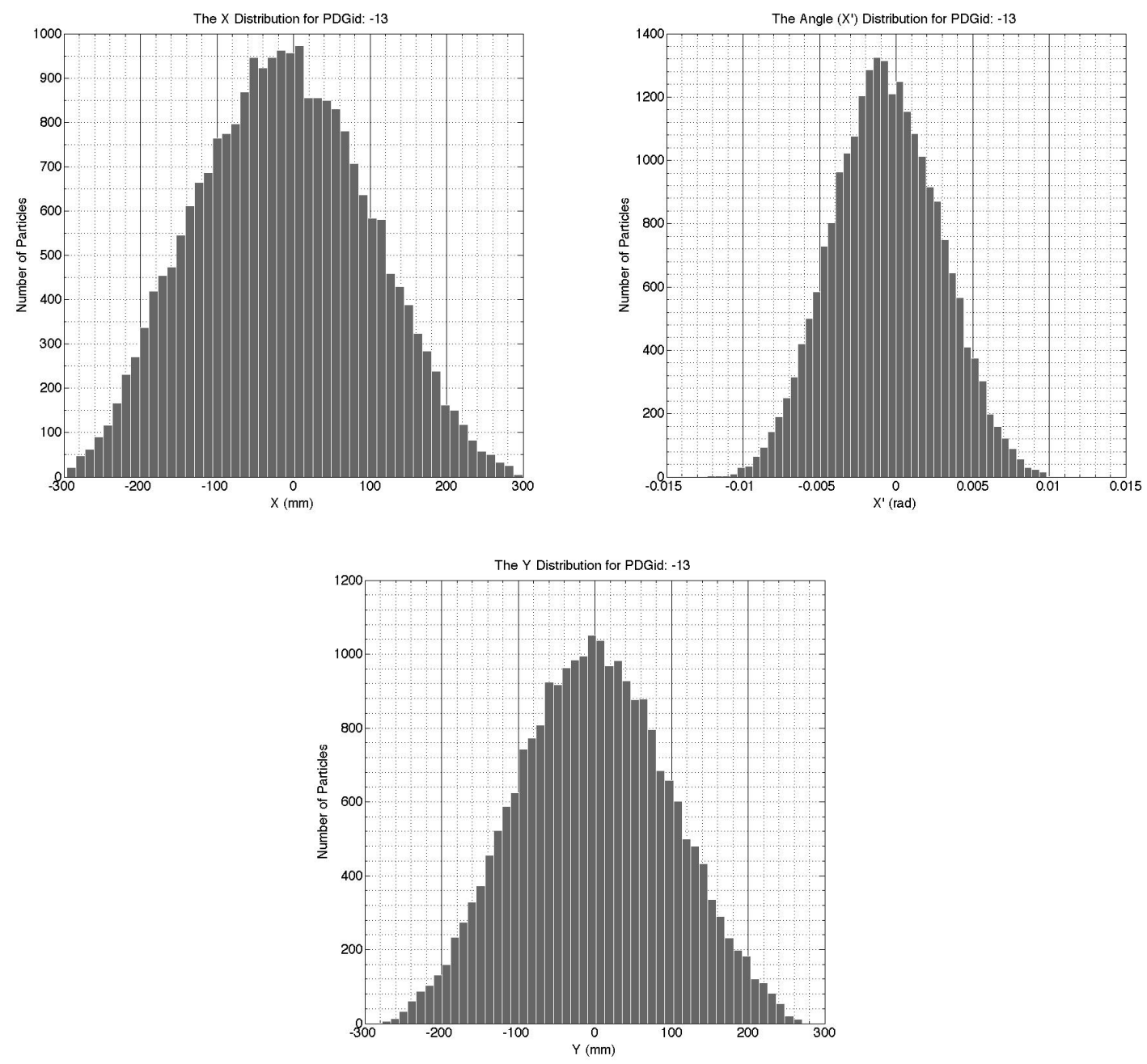

Figure A.5: Projections of distributions of $3.8 \pm 10 \% \mathrm{GeV} / \mathrm{c}$ muons onto x axis (upper left), $\mathrm{x}^{\prime}$ axis (upper right) and y axis (lower) 


\section{Acknowledgments}

The authors want to thank all the APC and nuSTORM colleagues for their continuous help in this design.

\section{References}

[1] D. Neuffer. Design considerations for a muon storage ring. Proc. Neutrino Mass Miniconference, Telemark, WI, 1980.

[2] A. Liu et al. $\nu$ storm facility design and simulation. Proceedings of IPAC2013, Shanghai, China, (MOODB203):55-57, 2013.

[3] P. Kyberd et al. Neutrinos from stored muons. Proposal to the Fermilab PAC, 2013.

[4] CERN. http://mad.web.cern.ch/mad/.

[5] V. Lebedev. http://www-bdnew.fnal.gov/pbar/organizationalchart/lebedev/ OptiM/optim.htm.

[6] T. Roberts. http://www. muonsinternal. com/muons3/G4beamline.

[7] Ao Liu. [csh script; version 1.1] convert sequence output from madx to optim under windows. http://frankliuao.com/research.html.

[8] Ao Liu. [csh script; version 1.06c] convert optim lattice to g4beamline input. http: //frankliuao.com/research.html. 